\title{
3 Research Square

\section{Triglyceride-glucose index in the development of peripheral artery disease: findings from the Atherosclerosis Risk in Communities (ARIC) Study}

Jing-Wei Gao

Sun Yat-Sen Memorial Hospital

Qing-Yun Hao

Sun Yat-Sen Memorial Hospital

Ming Gao

Sun Yat-Sen Memorial Hospital

Kun Zhang

Sun Yat-Sen Memorial Hospital

Xiong-Zhi Li

Sun Yat-Sen Memorial Hospital

Jing-Feng Wang

Sun Yat-Sen Memorial Hospital

Dominique A. Vuitton

Burgundy Franche-Comté University: Universite Bourgogne Franche-Comte

Shao-Ling Zhang

Sun Yat-Sen Memorial Hospital

Pin-Ming Liu ( $D$ liupm@mail.sysu.edu.cn )

Sun Yat-Sen Memorial Hospital

\section{Research Article}

Keywords: Triglyceride-glucose index, Insulin resistance, Peripheral artery disease, Risk factors, Cardiovascular disease

Posted Date: May 11th, 2021

DOI: https://doi.org/10.21203/rs.3.rs-496937/v1

License: (c) (1) This work is licensed under a Creative Commons Attribution 4.0 International License. Read Full License 


\section{Abstract \\ Background}

It remains unclear whether triglyceride-glucose (TyG) index, a surrogate marker of IR, was prospectively associated with incident PAD.

\section{Methods}

We included 12573 ARIC (Atherosclerosis Risk in Communities Study) participants free of PAD at baseline (1987-1989). The TyG index was determined using $\ln ($ fasting triglycerides $[\mathrm{mg} / \mathrm{dL}] \times$ fasting glucose $[\mathrm{mg} / \mathrm{dL}] / 2)$, and measured during 5 visits between 1987 and 2013. Incident PAD was defined as the first hospitalization with PAD diagnosis. We quantified the association of both baseline and trajectories of TyG index with incident PAD using Cox regression and logistic regression analysis, respectively.

\section{Results}

Over a median follow-up of 23 years, there were 1331 cases of incident PAD. After adjustment for traditional PAD risk factors, each 1-SD (0.58) increase in TyG index was associated with an $18.9 \%$ higher risk of incident PAD (hazard ratio, 1.189 [95\% $\mathrm{Cl}, 1.106-1.278]$ ). Results were similar when individuals were categorized by TyG index quartiles (hazard ratio, 1.363 [95\% $\mathrm{Cl}, 1.125-1.652]$; comparing extreme quartiles). Four distinct trajectories of TyG index were identified (low-increasing [43.0\%], moderate-stable [22.3\%], moderate-decreasing [27.6\%], and high-decreasing [7.1\%]). Trajectories of elevated TyG index levels had greater incident PAD after multivariable adjustment for potential cardiovascular risks. Compared with moderate-stable group (reference), high-decreasing group was associated with the highest risk of future incident PAD (odds ratio, $2.314[95 \% \mathrm{Cl}, 1.687-3.175]$ ).

\section{Conclusion}

Higher TyG index is independently associated with incident PAD. Long-term trajectories of TyG index help identify individuals at a higher risk of future PAD who deserve appropriate follow-up to detect asymptomatic disease.

\section{Background}

Lower extremity peripheral artery disease (PAD) is an important manifestation of systemic atherosclerosis affecting an estimated over 200 million people worldwide [1, 2]. It is associated with significant cardiovascular morbidity and mortality, with a variable spectrum of symptoms from none to severe when patients present with claudication or critical limb ischemia [3]. Despite its high prevalence 
and well-described adverse outcomes, the pathobiology of PAD is incompletely understood. There is a need to identify potential biomarkers that could predict the risk of PAD, and guide targeted screening to facilitate timely intervention at early stages of atherosclerosis.

Insulin resistance (IR), a pathophysiological state characterized by the attenuated insulin sensitivity of peripheral tissues, is the key feature of metabolic syndrome and type 2 diabetes [4]; and contributes significantly to the development of atherosclerotic cardiovascular disease [5]. However, the role of IR in PAD has been inadequately explored, compared with that of other atherogenic mechanisms such as inflammation [6-8]. Moreover, risk factors for PAD have not been as thoroughly investigated as those for coronary artery disease [9]. The triglyceride glucose (TyG) index, which is calculated using fasting triglycerides and fasting glucose, is a reliable measure of IR $[10,11]$. Growing evidence has demonstrated that the TyG index is related to morbidity and mortality of cardiovascular disease in the general population, patients with and those without diabetes $[12,13]$ This is possibly because elevated TyG index by itself contributes to systemic arterial atherosclerosis, including carotid atherosclerosis and coronary artery calcification, an established marker of subclinical atherosclerosis [14, 15]. However, long-term specific prospective studies on the relationship between the TyG index and PAD have not been performed.

We hypothesized that dynamic changes over the decades in IR, either improved or exacerbated, might modify the development of PAD. Accordingly, we used the data from the Atherosclerosis Risk in Communities (ARIC) study to evaluate the association of the TyG index with PAD and to determine the influence of baseline TyG index and different trajectories of its change over 20 years on the development of PAD.

\section{Methods}

\section{Study population}

The ARIC Study is a prospective cohort study that enrolled 15792 participants aged 45 to 64 years from 4 US communities (Forsyth County, North Carolina; Jackson, Mississippi; eight northern suburbs of Minneapolis, Minnesota; and Washington County, Maryland), aimed at investigating the natural history, etiology, and clinical manifestations of atherosclerotic disease in black and white men and women. The participants were recruited between 1987 to 1989 (visit 1). Four subsequent visits were conducted: visit 2 (1990-1992), visit 3 (1993-1995), visit 4 (1996-1998), and visit 5 (2011-2013). The details about the study design have been previously described [16]. Written informed consent was obtained from all ARIC participants, and the ARIC study was approved by the institutional review boards at each site.

We excluded participants who had PAD diagnosis at baseline $(n=613)$; those who had missing data regarding PAD ( $n=555)$; and those who had missing data regarding other covariates of interest $(n=1785)$. We also excluded participants who had no follow-up information on PAD $(n=266)$. This resulted in a final sample of 12573 participants for the analysis of association between baseline TyG index and incident PAD. We further excluded those participants with fewer than three valid TyG index during follow-up visits; 
the remaining 9296 participants were included in the analysis of association between TyG index groupbased trajectory and incident PAD (Figure 1).

\section{Data collection at baseline}

Trained interviewers collected information using standardized questionnaires on demographic, lifestyle, and detailed medical information at visit 1 . Age, sex, race, educational level, physical activity, alcohol consumption, and smoking status were self-reported. Educational attainment was categorized as basic (less than high school), intermediate (high school graduate or vocational school), and advanced (college, graduate school, or professional school). Smoking and alcohol drinking status were classified as current, former, or never. Body mass index (BMI) was calculated as weight in kilograms divided by height in meters squared. Seated blood pressure represented the mean of the last two of three measurements using a random-zero sphygmomanometer after a 5-minute rest. Hypertension was defined as systolic blood pressure readings $\geq 140 \mathrm{mmHg}$ or diastolic blood pressure readings $\geq 90 \mathrm{mmHg}$, or use of antihypertensive drugs in the previous two weeks. Diabetes was defined as fasting glucose level $\geq 126$ $\mathrm{mg} / \mathrm{dL}$ ( $\geq 7.0 \mathrm{mmol} / \mathrm{L}$ ), non-fasting glucose level $\geq 200 \mathrm{mg} / \mathrm{dL}$ ( $\geq 11.1 \mathrm{mmol} / \mathrm{L})$, self-reported physician diagnosis of diabetes, or use of antidiabetic drugs. Prevalent cardiovascular diseases such as coronary heart disease and stroke were determined according to both participants' self-report and measurements at visit 1 . Blood samples were obtained from participants who were asked to fast for 12 hours and stored at $-70^{\circ} \mathrm{C}$ according to standardized protocols until laboratory analysis [17]. Plasma total cholesterol, highdensity lipoprotein (HDL) cholesterol, and triglycerides were measured using automated enzymatic procedures, and low-density lipoprotein (LDL) cholesterol was calculated using the Friedewald equation when the concentration of triglycerides is $<400 \mathrm{mg} / \mathrm{dL}$ [18]. Estimated glomerular filtration rate (eGFR) was calculated using the Chronic Kidney Disease Epidemiology Collaboration creatinine equation [19]. Medications were determined through self-reported usage in the previous two weeks and inspection of medication containers that participants brought to the visit.

The triglyceride-glucose (TyG) index was calculated as $\ln$ (fasting triglycerides [mg/dL] $x$ fasting glucose $[\mathrm{mg} / \mathrm{dL}] / 2)$. Group-based trajectory analysis is designed to identify clusters of individuals with similar patterns of change over time. Trajectory groups were identified and then qualitatively examined and named to describe a visual pattern of change.

\section{Ascertainment of incident PAD}

Based on previous literature [8, 20, 21], PAD-related hospitalizations were identified by the following International Classification of Diseases Ninth Revision (ICD-9) discharge codes: peripheral vascular disease, unspecified (443.9); atherosclerosis of native arteries of the extremities, unspecified (440.20); atherosclerosis of native arteries of the extremities with intermittent claudication (440.21); atherosclerosis of native arteries of the extremities with rest pain (440.22); atherosclerosis of native arteries of the extremities with ulceration (440.23); atherosclerosis of native arteries of the extremities with gangrene (440.24); other atherosclerosis of native arteries of the extremities (440.29); atherosclerosis of bypass graft of the extremities (440.3); chronic total occlusion artery extremities 
(440.4); atherosclerosis of other specified arteries (440.8); coexisting leg amputation (84.11, 84.12, 84.15, 84.17); leg artery revascularization $(38.18,39.25,39.29,39.50)$; lower extremity ulcer and gangrene (707.1x). Incident PAD was defined as if a documented PAD-related hospitalization or a measured anklebrachial index $<0.90$ during follow-up visits in patients not diagnosed with PAD at visit 1 [3]. Critical limb ischemia (CLI), the severe form of PAD, was based on the discharge codes $(84.11,84.12,84.15,84.17$, 707.1x).

\section{Statistical Analysis}

Normally distributed continuous data were expressed as mean $\pm S D$, and the non-normally distributed continuous data, otherwise, were expressed as the median (interquartile range). Categorical data were expressed as numbers (percentage). Differences among groups were evaluated using analysis of variance (ANOVA) or Kruskal-Wallis $h$-test when appropriate for the continuous variables, and the $\chi^{2}$ test for the categorical variables. The follow-up period was set as the time from visit 1 (baseline) to the incident of PAD, or loss to follow-up, or September 30, 2015, whichever came first. Kaplan-Meier estimates were used to compute cumulative incidence of incident PAD by TyG index quartiles and the differences in estimates were compared using the log-rank procedure. Cox proportional hazards regression model was used to calculate hazard ratios and 95\% Cls between TyG index and time to incident PAD. Three multivariate models with progressive degrees of adjustment were used to adjust for potential confounders. Model 1 adjusted for age, sex, and race. Model 2 further adjusted for antihypertensive drugs, body mass index, coronary heart disease, cholesterol-lowering drugs, diastolic blood pressure, diabetes, drinking status, education level, hypertension, physical activity during leisure time, systolic blood pressure, smoking status, sport during leisure time, stroke. Model 3 was additionally further adjusted for eGFR, insulin, LDL cholesterol and total cholesterol. We further used a restricted cubic spline regression model with 3 knots to assess the nonlinear dose-response association between TyG index and incident PAD. Subgroup analyses were performed stratifying by age, sex, race, smoking status, body mass index, hypertension, and diabetes at baseline, respectively. Moreover, we used latent class models to identify different patterns of longitudinal change in TyG index during follow-up. Models were fit using R 3.6.1 based on R package tidyLPA. Group-based trajectory analysis was designed to identify clusters of individuals with similar patterns of change over time [22]. The optimal number of trajectory groups was determined using a combination of Bayesian information criterion and number of observations in each group. Participants were assigned to the trajectory group for which they had the greatest posterior predictive probability. To estimate the association of trajectory groups with incident PAD, trajectory groups was included as an independent variable in a logistic regression model examining predictors of follow-up incident PAD.

All analyses were conducted in SPSS version 23 (SPSS, Inc, Chicago, Illinois). A two-sided $P$ value of < 0.05 was considered statistically significant.

\section{Results}




\section{Baseline characteristics according to quartiles of TyG index}

The average age of all the participants was $54.2 \pm 5.7$ years, $5784(46.0 \%)$ were men, $3202(25.5 \%)$ were current smokers, and 5298 (42.1\%) were hypertensive. The mean TyG index was $8.7 \pm 0.6$ (Table I in the Data Supplement). We categorized the included population into four groups based on the quartiles of baseline TyG index (Table 1). Participants with a higher TyG index were older, male, and white; had higher levels of body mass index, systolic and diastolic blood pressures, total cholesterol, LDL cholesterol, triglycerides, insulin and fasting plasma glucose; had lower rates of current drinkers, lower levels of education, physical activity score, HDL cholesterol, and eGFR. Likewise, participants in a higher TyG index quartile had a higher prevalence of hypertension, diabetes, coronary heart disease, stroke; and were more prone to take antihypertensive drugs, and cholesterol-lowering drugs. Compared with the analytic cohort, excluded participants were more likely to be female, black, current smokers and non-current drinkers; have lower levels of education, physical activity score and HDL cholesterol; have higher levels of body mass index, systolic and diastolic blood pressures, fasting plasma glucose, insulin, total cholesterol, LDL cholesterol, triglycerides, and eGFR, have correspondingly higher rates of hypertension, diabetes, coronary heart disease, stroke (Table I in the Data Supplement).

\section{Association between baseline TyG index and incident PAD}

During a follow-up period of $23.0(14.7,25.5)$ years, 1331 incident cases $(10.6 \%)$ of PAD were observed. As Table 1 shows, the risk of incident PAD increased with increasing quartiles of TyG index (quartiles 1-4: 228 [7.3\%] versus 287 [9.1\%] versus 369 [11.7\%] versus 447 [14.2\%]; $P$ for trend<0.001). A similar trend in prevalence of incident CLI was observed. Both unadjusted and adjusted models yielded identical results. The highest risk of incident PAD was found in participants in the highest TyG index quartile (Table 2). In the multivariate model that measured TyG index as a continuous variable, a 1-SD increase (corresponding to 0.58 ) in TyG index was associated with an $18.9 \%$ higher risk of incident PAD (hazard ratio, 1.189 [95\% $\mathrm{Cl}, 1.106,1.278] ; P<0.001 ;$ Table 2). Results were similar when we categorized individuals by TyG index quartiles: the highest risk of incident PAD was observed in the participants with the highest TyG index, in both unadjusted and adjusted models $(P<0.05$, Table 2$)$. In the final model, the hazard ratios $(95 \% \mathrm{Cls})$ for incident PAD comparing the second, third, fourth quartiles of TyG index to the first quartile were 1.059 (95\% Cl, 0.888-1.264), 1.224 (95\% Cl, 1.029-1.457), and 1.363 (95\% Cl, 1.125-1.652), respectively (Table 2; Figure 2). Figure 3 shows the restricted cubic splines of the risk of incident PAD across levels of TyG index. Consistent with the analysis using quartiles of sample distribution, the risk of incident PAD increased in participants with a higher TyG index. However, there was no significant difference for risk of incident PAD in participants with TyG index $<8.6$ (Figure 3).

When participants were stratified by age ( $\leq 54$ or $>54$ years), sex (male or female), race (white or black), smoking status (current, former or never), body mass index ( $<30$ or $\geq 30 \mathrm{~kg} / \mathrm{m}^{2}$ ), hypertension (yes or no), and diabetes (yes or no), the association between TyG index and incident PAD remained consistent (Figure 4). 


\section{Association between TyG index trajectories and incident PAD}

9296 participants were included for further trajectory analysis (Figure 1), and four discrete trajectories in TyG index from visit 1 to visit 5 were identified (Figure 5); i.e., $43.0 \%$ of the cohort $(n=3998)$ had long-term low TyG index values, with a slight increase in later life (low-increasing), 22.3\% ( $n=2075)$ maintained fairly stable moderate TyG index values all along the follow-up (moderate-stable), $27.6 \%(n=2567)$ had long-term moderate TyG index values with a slight decrease in later life (moderate-decreasing), and 7.1\% $(n=656)$ had long-term high TyG index values with a slight decrease in later life (high-decreasing) throughout the follow-up. As shown in Figure 6, the prevalence of incident PAD was 7.8\%, 10.3\%, 13.9\% and $20.9 \%$ 冈in moderate-stable, low-increasing, moderate-decreasing and high-decreasing TyG index groups, respectively ( $P$ for trend=0.001). In multivariable analysis, compared with moderate-stable, the odds ratios (95\% Cls) for low-increasing, moderate-decreasing, and high-decreasing were $1.118(95 \% \mathrm{Cl}$, 0.914-1.368), 1.507 (95\% Cl, 1.202-1.890), and 2.314 (95\% Cl, 1.687-3.175), respectively (Table 3).

\section{Discussion}

In this large-scale, community-based prospective cohort of middle-aged adults, we show for the first time that higher levels of TyG index are significantly associated with an increased risk of PAD over a median follow-up of 23 years. Furthermore, we identify that four distinct trajectories of TyG index confer different risk of PAD, and two-decade trajectory with elevated TyG index carries greater risk of future incident PAD. These findings suggest a potential role for long-term trajectory with high IR in the pathogenesis of PAD.

IR has been considered as an important risk factor for cardiovascular disease [23, 24]. Methods to directly measure IR are invasive, complex, and costly $[10,11]$. Therefore, a number of surrogate markers of IR have been proposed and compared with the gold standard of the hyperinsulinemic-euglycemic clamp [25]. Homeostatic model assessment of IR (HOMA-IR), which is calculated by fasting insulin and glucose, is commonly used for assessing IR. However, the insulin concentrations are not routinely measured in clinical settings. As for the ARIC cohort, there were many missing insulin values due to low detection rate and absence of no insulin measurement at visit 2 and visit 3. Therefore, in this large-scale, communitybased prospective cohort study, we utilized the TyG index as a biomarker of IR. The TyG index has been proved to be highly correlated with the euglycemic-hyperinsulinemic clamp test [10], and thus has a validity similar to HOMA-IR [11]. The immense advantage of using such a simple method of IR identification is obviously that it is easily accessible in any clinical settings, making our findings immediately usable by clinicians.

Among the multiple pathological consequences of atherosclerosis, PAD has generally been paid far less attention than coronary diseases or stroke. Based on the updated estimates of PAD prevalence at global regional levels in 2015, 236.62 million (5.56\%) people aged 25 years and older had PAD, among whom $73 \%$ were in low-income and middle-income countries [26]. However, only about $10 \%$ of patients with PAD demonstrate the typical symptomatology of intermittent claudication; and the majority of patients with PAD are thus asymptomatic and underdiagnosed [27]. Therefore, it is of great importance to regularly 
measure markers of risk for PAD and take preventive measures at an earlier clinical stage. In addition to age, significant atherosclerotic risk factors for PAD include cigarette smoking, dyslipidemia, and diabetes [28]. It has been well-established that IR and coexisting hyperinsulinemia are implicated in the development of dyslipidemia, hypertension, hypercoagulability, and atherosclerosis [29,30]. However, there is a paucity of prospective data regarding the association between IR assessed by HOMA-IR and PAD [31, 32]. A cross-sectional study of 3242 adults from data in the National Health and Nutrition Examination Survey has identified a positive association between IR and PAD [31]. Only one communitybased longitudinal study, which enrolled 4208 participants over the age of 65 years in the Cardiovascular Health Study, showed that IR was associated with a higher risk of clinical PAD [32]. In line with previous studies, our study of larger sample size showed that the metabolic risk factors such as hypertension, diabetes, and hyperlipidemia, were more obvious with higher quartiles of TyG index. Meanwhile, individuals in the highest quartile of baseline TyG index had a 2.15-fold higher risk of PAD incidence compared to those with the lowest quartile. After adjusting all the aforementioned PAD risk factors, this positive association attenuated but remained significant. These findings suggested that clinical management of TyG index may bring additional effect on PAD development even under vigorous control of traditional risk factors. Further studies are needed to unravel this aspect. Most previous studies based on the TyG index measured at a single time point, which may not reflect long-term exposure because of variation of the TyG index level over time $[12,13]$. Therefore, measurements of long-term trajectories of TyG index provide more reliable and robust results. Our study is the first, to our knowledge, to investigate the impact of long-term patterns of IR assessed by TyG index on future PAD incidence. Latent class modeling, as used in our analyses, has allowed identification of different patterns of IR changes as separate trajectory groups, which provides a more realistic understanding of long-term trends in IR as compared to population mean levels. Data from the Coronary Artery Risk Development in Young Adults (CARDIA) Study involving 2414 individuals showed that high-increasing IR (assessed by HOMA-IR) trajectory over 25 years was associated with impaired diastolic function compared with low-decreasing IR trajectory [33]. Another post-analyses by using CARDIA Study data also demonstrated that highincreasing HOMA-IR (referent: low-stable) was associated with greater prevalent diabetes in participants with non-alcoholic fatty liver disease [34]. We highlighted the fact that within the ARIC population there were heterogeneous patterns of trends in TyG index. Our results further suggest that those trajectory groups with long-term moderate and high TyG indexes beginning in midlife are at a greater risk of incident PAD over 20 years, despite the minor reduction of TyG index observed in later life.

Higher TyG index, a surrogate marker of $I R$, which induce an imbalance in glucose metabolism that generates chronic hyperglycemia, and can also alter lipid metabolism and lead to dyslipidemia [35]. Although the potential mechanisms responsible for the association of the TyG index with the risk of PAD have not been elucidated, our findings at least in part support the important role that metabolic disturbances, including hyperglycemia and hypertriglyceridemia, in the pathophysiology of PAD [36]. Many studies have indicated that IR could promote atherosclerosis not only through mechanisms that involve systemic factors, such as dyslipidemia, hypertension, and a proinflammatory state, but also 
through the effect of perturbed insulin signaling at the level of the intimal cells [30,37]. Therefore, further studies are warranted to elucidate the precise mechanism for the association.

\section{Study Limitations}

Several limitations of this investigation are worth noting. The study included only whites and blacks aged 45-64 years at baseline, results may differ outside this age range and in other ethnicities. Due to the shortage of records on insulin in the ARIC study, we cannot compare trajectories of TyG index with HOMAIR for predicting incident PAD. Similarly, because of the missing data of endothelial function, we cannot explore the potential role of endothelial dysfunction in the association between TyG index and PAD, which was demonstrated to be at least one possible biological pathway between IR and atherosclerosis [38, 39]. Moreover, as for the nature of any observational studies, we cannot exclude the possibility of residual confounders despite our careful adjustment for the well-known and suspected risk factors.

\section{Conclusion}

Higher TyG index is associated with incident PAD, which is independent of other traditional atherosclerotic risk factors; suggesting that metabolic dysfunction is actually involved in the pathophysiology of PAD. Trajectories denoting long-term exposure to high IR assessed by TyG index provide additional information about the cumulative burden of risk for future PAD.

\section{Abbreviations}

ARIC: Atherosclerosis Risk in Communities Study; CARDIA: Coronary Artery Risk Development in Young Adults; CLI: critical limb ischemia; eGFR: estimated glomerular filtration rate; HDL: high-density lipoprotein; HOMA-IR: homeostatic model assessment of insulin resistance; IR: insulin resistance; LDL: low-density lipoprotein; PAD: peripheral artery disease; TyG: triglyceride-glucose.

\section{Declarations}

\section{Authors' contributions}

JWG, QYH and PML contributed to the study concept, and drafted the manuscript. JWG and QYH has full access to the data and performed the analyses; JWG and QYH assisted in data interpretation; MG, KZ and XZL helped in the data methods and presentation; JWG, DAV, SLZ and PML critically revised the manuscript. JFW and PML supervised the study analyses. All authors read and approved the final manuscript

\section{Acknowledgments}

We thank the investigators, the staffs and participants of the ARIC Study for their highly valued contributions. 


\section{Funding}

This work was supported by grants from National Natural Science Foundation of China [81900379, $81870315,81970683]$. The ARIC Study has been funded in whole or in part with Federal funds from the National Heart, Lung, and Blood Institute, National Institutes of Health, Department of Health and Human Services, under Contract Nos. (HHSN268201700001I, HHSN268201700003I, HHSN268201700005I, HHSN268201700004I, HHSN2682017000021).

\section{Availability of data and materials}

The data that support the findings of this study are available from the corresponding author upon reasonable request.

\section{Ethics approval and consent to participate}

The study was approved by the institutional review boards at all field centers of ARIC study, and informed consent was obtained from all participants.

\section{Consent for publication}

The consent to publish was obtained from all participants in this study.

\section{Competing interests}

The authors declare that they have no competing interests.

\section{Author details}

${ }^{1}$ Department of Cardiology, Sun Yat-sen Memorial Hospital of Sun Yat-sen University, Guangzhou, China.

${ }^{2}$ Department of Radiology, Sun Yat-sen Memorial Hospital of Sun Yat-sen University, Guangzhou, China.

${ }^{3}$ EA3181, Université Bourgogne Franche-Comté, Besançon, France. ${ }^{4}$ Department of Endocrinology, Sun Yat-sen Memorial Hospital of Sun Yat-sen University, Guangzhou, China.

\section{References}

1. Fowkes FG, Rudan D, Rudan I, Aboyans V, Denenberg JO, McDermott MM, Norman PE, Sampson UK, Williams LJ, Mensah GA, et al. Comparison of global estimates of prevalence and risk factors for peripheral artery disease in 2000 and 2010: a systematic review and analysis. Lancet. 2013; 382(9901):1329-40.

2. Criqui MH, Aboyans V. Epidemiology of peripheral artery disease. Circ Res. 2015;116(9):1509-26.

3. Gerhard-Herman MD, Gornik HL, Barrett C, Barshes NR, Corriere MA, Drachman DE, Fleisher LA, Fowkes FG, Hamburg NM, Kinlay S, et al. 2016 AHA/ACC Guideline on the management of patients with lower extremity peripheral artery disease: executive summary: a report of the American College 
of Cardiology/American Heart Association Task Force on Clinical Practice Guidelines. Circulation. 2017;135(12):e686-e725.

4. Faerch $K$, Vaag A, Holst JJ, Hansen T, Jørgensen T, Borch-Johnsen K. Natural history of insulin sensitivity and insulin secretion in the progression from normal glucose tolerance to impaired fasting glycemia and impaired glucose tolerance: the Inter99 study. Diabetes Care. 2009;32(3):439-44.

5. DeFronzo RA. Insulin resistance, lipotoxicity, type 2 diabetes and atherosclerosis: the missing links. The Claude Bernard Lecture 2009. Diabetologia. 2010;53(7):1270-87.

6. Brevetti G, Giugliano G, Brevetti L, Hiatt WR. Inflammation in peripheral artery disease. Circulation. 2010;122(18):1862-75.

7. Dann R, Hadi T, Montenont E, Boytard L, Alebrahim D, Feinstein J, Allen N, Simon R, Barone K, Uryu K, et al. Platelet-derived MRP-14 induces monocyte activation in patients with symptomatic peripheral artery disease. J Am Coll Cardiol. 2018;71(1):53-65.

8. Ding N, Yang C, Ballew SH, Kalbaugh CA, McEvoy JW, Salameh M, Aguilar D, Hoogeveen RC, Nambi V, Selvin E, et al. Fibrosis and inflammatory markers and long-term risk of peripheral artery disease: the ARIC Study. Arterioscler Thromb Vasc Biol. 2020;40(9):2322-31.

9. Kullo IJ, Leeper NJ. The genetic basis of peripheral arterial disease: current knowledge, challenges, and future directions. Circ Res. 2015;116(9):1551-60.

10. Guerrero-Romero F, Simental-Mendía LE, González-Ortiz M, Martínez-Abundis E, Ramos-Zavala MG, Hernández-González SO, Jacques-Camarena O, Rodríguez-Morán M. The product of triglycerides and glucose, a simple measure of insulin sensitivity. Comparison with the euglycemic-hyperinsulinemic clamp. J Clin Endocrinol Metab. 2010;95(7):3347-51.

11. Simental-Mendía LE, Rodríguez-Morán M, Guerrero-Romero F. The product of fasting glucose and triglycerides as surrogate for identifying insulin resistance in apparently healthy subjects. Metab Syndr Relat Disord. 2008;6(4):299-304.

12. Ma X, Dong L, Shao Q, Cheng Y, Lv S, Sun Y, Shen H, Wang Z, Zhou Y, Liu X. Triglyceride glucose index for predicting cardiovascular outcomes after percutaneous coronary intervention in patients with type 2 diabetes mellitus and acute coronary syndrome. Cardiovasc Diabetol. 2020(1);19:31.

13. Barzegar N, Tohidi M, Hasheminia M, Azizi F, Hadaegh F. The impact of triglyceride-glucose index on incident cardiovascular events during 16 years of follow-up: Tehran Lipid and Glucose Study. Cardiovasc Diabetol. 2020 29;19:155.

14. Irace C, Carallo C, Scavelli FB, De Franceschi MS, Esposito T, Tripolino C, Gnasso A. Markers of insulin resistance and carotid atherosclerosis. A comparison of the homeostasis model assessment and triglyceride glucose index. Int J Clin Pract. 2013;67(7):665-72.

15. Park K, Ahn CW, Lee SB, Kang S, Nam JS, Lee BK, Kim JH, Park JS. Elevated TyG index predicts progression of coronary artery calcification. Diabetes Care. 2019;42(8):1569-73.

16. The Atherosclerosis Risk in Communities (ARIC) Study: design and objectives. The ARIC investigators. Am J Epidemiol. 1989;129(4):687-702. 
17. Papp AC, Hatzakis H, Bracey A, Wu KK. ARIC hemostasis study--I. Development of a blood collection and processing system suitable for multicenter hemostatic studies. Thromb Haemost. 1989;61(1):15-9.

18. Friedewald WT, Levy RI, Fredrickson DS. Estimation of the concentration of low-density lipoprotein cholesterol in plasma, without use of the preparative ultracentrifuge. Clin Chem. 1972;18(6):499-502.

19. Levey AS, Stevens LA, Schmid CH, Zhang YL, Castro AF 3rd, Feldman HI, Kusek JW, Eggers P, Van Lente $F$, Greene T, et al. A new equation to estimate glomerular filtration rate. Ann Intern Med. 2009;150(9):604-12.

20. Wattanakit K, Folsom AR, Selvin E, Coresh J, Hirsch AT, Weatherley BD. Kidney function and risk of peripheral arterial disease: results from the Atherosclerosis Risk in Communities (ARIC) Study. J Am Soc Nephrol. 2007;18(2):629-36.

21. Liang J, Zhang H, Sun X, Liao L, Li X, Hu X, Du J, Zhuang X, Liao X. Association between calf girth and peripheral artery disease in the Atherosclerosis Risk in Communities Study. J Cardiol. 2020;76(3):273-9.

22. Nagin DS, Odgers CL. Group-based trajectory modeling in clinical research. Annu Rev Clin Psychol. 2010;6:109-38.

23. Laakso M, Kuusisto J. Insulin resistance and hyperglycaemia in cardiovascular disease development. Nat Rev Endocrinol. 2014;10(5):293-302.

24. Adeva-Andany MM, Martínez-Rodríguez J, González-Lucán M, Fernández-Fernández C, CastroQuintela E. Insulin resistance is a cardiovascular risk factor in humans. Diabetes Metab Syndr. 2019;13(2):1449-55.

25. Bastard JP, Lavoie ME, Messier V, Prud'homme D, Rabasa-Lhoret R. Evaluation of two new surrogate indices including parameters not using insulin to assess insulin sensitivity/resistance in non-diabetic postmenopausal women: a MONET group study. Diabetes Metab. 2012;38(3):258-63.

26. Song P, Rudan D, Zhu Y, Fowkes FJI, Rahimi K, Fowkes FGR, Rudan I. Global, regional, and national prevalence and risk factors for peripheral artery disease in 2015: an updated systematic review and analysis. Lancet Glob Health. 2019;7(8):e1020-30.

27. Hirsch AT, Criqui MH, Treat-Jacobson D, Regensteiner JG, Creager MA, Olin JW, Krook SH, Hunninghake DB, Comerota AJ, Walsh ME, et al. Peripheral arterial disease detection, awareness, and treatment in primary care. JAMA. 2001;286(11):1317-24.

28. Barnes JA, Eid MA, Creager MA, Goodney PP. Epidemiology and risk of amputation in patients with diabetes mellitus and peripheral artery disease. Arterioscler Thromb Vasc Biol. 2020;40(8):1808-17.

29. Wang T, Zhao Z, Xu Y, Qi L, Xu M, Lu J, Li M, Chen Y, Dai M, Zhao W, et al. Insulin resistance and $\beta$-cell dysfunction in relation to cardiometabolic risk patterns. J Clin Endocrinol Metab. 2018;103(6):220715.

30. Bornfeldt KE, Tabas I. Insulin resistance, hyperglycemia, and atherosclerosis. Cell Metab. 2011;14(5):575-85. 
31. Pande RL, Perlstein TS, Beckman JA, Creager MA. Association of insulin resistance and inflammation with peripheral arterial disease: the National Health and Nutrition Examination Survey, 1999 to 2004. Circulation. 2008;118(1):33-41.

32. Britton KA, Mukamal KJ, Ix JH, Siscovick DS, Newman AB, de Boer IH, Thacker EL, Biggs ML, Gaziano JM, Djoussé L. Insulin resistance and incident peripheral artery disease in the Cardiovascular Health Study. Vasc Med. 2012;17(2):85-93.

33. Gidding SS, Jacobs DR Jr, Kishi S, Lima J, Lloyd-Jones D, Reis JP, Schreiner PJ, Zmora R, Allen NB. Fasting glucose and insulin resistance trajectories during young adulthood and mid-life cardiac structure and function. J Diabetes Complications. 2019;33(5):356-62.

34. VanWagner LB, Ning H, Allen NB, Siddique J, Carson AP, Bancks MP, Lewis CE, Carr JJ, Speliotes E, Terrault NA, et al. Twenty-five-year trajectories of insulin resistance and pancreatic $\beta$-cell response and diabetes risk in nonalcoholic fatty liver disease. Liver Int. 2018;38(11):2069-81.

35. Ferrannini E, Barrett EJ, Bevilacqua S, DeFronzo RA. Effect of fatty acids on glucose production and utilization in man. J Clin Invest. 1983;72(5):1737-47.

36. Garg PK, Biggs ML, Carnethon M, Ix JH, Criqui MH, Britton KA, Djoussé L, Sutton-Tyrrell K, Newman $A B$, Cushman $M$, et al. Metabolic syndrome and risk of incident peripheral artery disease: the cardiovascular health study. Hypertension. 2014;63(2):413-9.

37. Giacco F, Brownlee M. Oxidative stress and diabetic complications. Circ Res. 2010;107(9):1058-70.

38. Kim JA, Montagnani M, Koh KK, Quon MJ. Reciprocal relationships between insulin resistance and endothelial dysfunction: Molecular and pathophysiological mechanisms. Circulation. 2006;113(15):1888-904.

39. Brevetti G, Schiano V, Chiariello M. Endothelial dysfunction: A key to the pathophysiology and natural history of peripheral arterial disease? Atherosclerosis. 2008;197(1):1-11.

\section{Tables}

Table 1. Baseline characteristics of study participants by quartiles of TyG index 


\begin{tabular}{|c|c|c|c|c|c|}
\hline Characteristics & $\begin{array}{l}\text { Quartile } 1 \\
(n=3143)\end{array}$ & $\begin{array}{l}\text { Quartile } 2 \\
(n=3145)\end{array}$ & $\begin{array}{l}\text { Quartile } 3 \\
(n=3142)\end{array}$ & $\begin{array}{l}\text { Quartile } 4 \\
(n=3143)\end{array}$ & $\begin{array}{l}P \\
\text { value }\end{array}$ \\
\hline TyG index & $8.0 \pm 0.2$ & $8.4 \pm 0.1$ & $8.8 \pm 0.1$ & $9.4 \pm 0.4$ & $<0.001$ \\
\hline Age, years & $53.1 \pm 5.7$ & $54.1 \pm 5.7$ & $54.7 \pm 5.7$ & $55.1 \pm 5.7$ & $<0.001$ \\
\hline Male, \% & $1171(37.3 \%)$ & $\begin{array}{l}1347 \\
(42.8 \%)\end{array}$ & $1565(49.8 \%)$ & $1701(54.1 \%)$ & $<0.001$ \\
\hline White, \% & $2187(69.6 \%)$ & $\begin{array}{l}2360 \\
(75.0 \%)\end{array}$ & $2473(78.7 \%)$ & $2500(79.5 \%)$ & $<0.001$ \\
\hline $\mathrm{BMI}, \mathrm{kg} / \mathrm{m}^{2}$ & $25.6 \pm 4.8$ & $27.0 \pm 5.1$ & $28.1 \pm 5.2$ & $29.7 \pm 5.1$ & $<0.001$ \\
\hline SBP, mmHg & $117.3 \pm 18.9$ & $119.3 \pm 18.4$ & $121.5 \pm 17.9$ & $125.1 \pm 18.3$ & $<0.001$ \\
\hline $\mathrm{DBP}, \mathrm{mmHg}$ & $72.4 \pm 11.5$ & $72.9 \pm 11.2$ & $73.8 \pm 10.9$ & $74.8 \pm 10.9$ & $<0.001$ \\
\hline Smoking status, \% & & & & & $<0.001$ \\
\hline Current smoker & $711(22.6 \%)$ & $846(26.9 \%)$ & $853(27.1 \%)$ & $792(25.2 \%)$ & \\
\hline Former smoker & $931(29.6 \%)$ & $992(31.5 \%)$ & $1052(33.5 \%)$ & $1175(37.4 \%)$ & \\
\hline Never smoker & $1501(47.8 \%)$ & $\begin{array}{l}1307 \\
(41.6 \%)\end{array}$ & $1237(39.4 \%)$ & $1176(37.4 \%)$ & \\
\hline Drinking status, \% & & & & & $<0.001$ \\
\hline Current drinker & $1873(59.6 \%)$ & $\begin{array}{l}1814 \\
(57.7 \%)\end{array}$ & $1767(56.2 \%)$ & $1736(55.2 \%)$ & \\
\hline Former drinker & $496(15.8 \%)$ & $569(18.1 \%)$ & $628(20.0 \%)$ & $664(21.1 \%)$ & \\
\hline Never drinker & $774(24.6 \%)$ & $762(24.2 \%)$ & $747(23.8 \%)$ & $743(23.6 \%)$ & \\
\hline Education level, \% & & & & & $<0.001$ \\
\hline Basic education & $601(19.1 \%)$ & $695(22.1 \%)$ & $701(22.3 \%)$ & $836(26.6 \%)$ & \\
\hline Intermediate education & $1220(38.8 \%)$ & $\begin{array}{l}1264 \\
(40.2 \%)\end{array}$ & $1351(43.0 \%)$ & $1334(42.4 \%)$ & \\
\hline Advanced education & $1322(42.1 \%)$ & $\begin{array}{l}1186 \\
(37.7 \%)\end{array}$ & $1090(34.7 \%)$ & $973(31.0 \%)$ & \\
\hline \multicolumn{6}{|l|}{ Physical activity score } \\
\hline $\begin{array}{l}\text { Sport during leisure } \\
\text { time }\end{array}$ & $2.5 \pm 0.8$ & $2.5 \pm 0.8$ & $2.5 \pm 0.8$ & $2.4 \pm 0.8$ & 0.002 \\
\hline $\begin{array}{l}\text { Physical activity during } \\
\text { leisure time }\end{array}$ & $2.4 \pm 0.6$ & $2.4 \pm 0.6$ & $2.4 \pm 0.6$ & $2.3 \pm 0.6$ & $<0.001$ \\
\hline Incident PAD, \% & $228(7.3 \%)$ & $287(9.1 \%)$ & $369(11.7 \%)$ & $447(14.2 \%)$ & $<0.001$ \\
\hline Incident CLI, \% & $22(0.7 \%)$ & $20(0.6 \%)$ & $41(1.3 \%)$ & $103(3.3 \%)$ & $<0.001$ \\
\hline
\end{tabular}




\begin{tabular}{|llllll|}
\hline Hypertension, \% & $979(31.1 \%)$ & $\begin{array}{l}1170 \\
(37.2 \%)\end{array}$ & $1370(43.6 \%)$ & $1779(56.6 \%)$ & $<0.001$ \\
\hline Diabetes, \% & $57(1.8 \%)$ & $129(4.1 \%)$ & $232(7.4 \%)$ & $984(31.3 \%)$ & $<0.001$ \\
\hline $\begin{array}{l}\text { Coronary heart disease, } \\
\%\end{array}$ & $67(2.1 \%)$ & $122(3.9 \%)$ & $175(5.6 \%)$ & $247(7.9 \%)$ & $<0.001$ \\
\hline Stroke, \% & $66(2.1 \%)$ & $67(2.1 \%)$ & $96(3.1 \%)$ & $123(3.9 \%)$ & $<0.001$ \\
\hline $\begin{array}{l}\text { Antihypertensive } \\
\text { medication, \% }\end{array}$ & $586(18.6 \%)$ & $800(25.4 \%)$ & $976(31.1 \%)$ & $1370(43.6 \%)$ & $<0.001$ \\
\hline $\begin{array}{l}\text { Cholesterol-lowering } \\
\text { medication, \% }\end{array}$ & $32(1.0 \%)$ & $77(2.4 \%)$ & $102(3.2 \%)$ & $140(4.5 \%)$ & $<0.001$ \\
\hline FPG, mg/dL & $94.2 \pm 8.6$ & $98.9 \pm 11.5$ & $103.7 \pm 17.7$ & $133.8 \pm 63.1$ & $<0.001$ \\
\hline Insulin, $\mu$ IU/mL & $6.0(4.0,9.0)$ & $8.0(6.0$, & $10.0(7.0$, & $14.0(10.0$, & $<0.001$ \\
\hline HDL-C, mg/dL & $62.9 \pm 17.7$ & $54.6 \pm 15.6$ & $47.8 \pm 13.9$ & $41.7 \pm 12.3$ & $<0.001$ \\
\hline LDL-C, mg/dL & $121.4 \pm 34.1$ & $136.3 \pm 37.4$ & $146.2 \pm 38.1$ & $145.5 \pm 40.4$ & $<0.001$ \\
\hline TC, mg/dL & $197.0 \pm 36.0$ & $209.8 \pm 38.0$ & $220.4 \pm 39.4$ & $229.4 \pm 43.3$ & $<0.001$ \\
\hline Triglycerides, mg/dL & $63.6 \pm 12.4$ & $94.7 \pm 13.2$ & $131.7 \pm 20.9$ & $211.2 \pm 62.8$ & $<0.001$ \\
\hline eGFR, mL/min/1.73m ${ }^{2}$ & $105.6 \pm 14.8$ & $102.5 \pm 14.5$ & $100.9 \pm 15.4$ & $99.9 \pm 16.5$ & $<0.001$ \\
\hline
\end{tabular}

Values are mean $\pm S D$ for normally distributed data and median and interquartile range for non-normally distributed data, or $\mathrm{n}(\%)$.

$B M /$ body mass index, $C L /$ critical limb ischemia; $D B P$ diastolic blood pressure, eGFR estimated glomerular filtration rate, $F P G$ fasting plasma glucose, $H D L-C$ high-density lipoprotein cholesterol, $L D L-C$ low-density lipoprotein cholesterol, $P A D$ peripheral artery disease, $S B P$ systolic blood pressure, $T C$ total cholesterol.

Table 2. Risk of incident PAD for baseline TyG index 


\begin{tabular}{|c|c|c|c|c|c|c|c|}
\hline \multirow{2}{*}{$\begin{array}{l}\text { TyG } \\
\text { index }\end{array}$} & \multirow{2}{*}{$\begin{array}{l}\text { Events/No. } \\
\text { at risk }\end{array}$} & Model 1 & \multirow{2}{*}{$\begin{array}{l}P \\
\text { value }\end{array}$} & Model 2 & \multirow{2}{*}{$\begin{array}{l}P \\
\text { value }\end{array}$} & Model 3 & \multirow{2}{*}{$\begin{array}{l}P \\
\text { value }\end{array}$} \\
\hline & & $\mathrm{HR}(95 \% \mathrm{Cl})$ & & $\mathrm{HR}(95 \% \mathrm{Cl})$ & & $\begin{array}{l}\mathrm{HR}(95 \% \\
\mathrm{Cl})\end{array}$ & \\
\hline $\begin{array}{l}\text { Quartile } \\
1\end{array}$ & $228 / 3143$ & Reference & 1.0 & Reference & 1.0 & Reference & 1.0 \\
\hline $\begin{array}{l}\text { Quartile } \\
2\end{array}$ & $287 / 3145$ & $\begin{array}{l}1.271 \\
(1.067 \\
1.513)\end{array}$ & 0.007 & $\begin{array}{l}1.110 \\
(0.931 \\
1.323)\end{array}$ & 0.246 & $\begin{array}{l}1.059 \\
(0.888, \\
1.264)\end{array}$ & 0.524 \\
\hline $\begin{array}{l}\text { Quartile } \\
3\end{array}$ & $369 / 3142$ & $\begin{array}{l}1.648 \\
(1.395, \\
1.946)\end{array}$ & $<0.001$ & $\begin{array}{l}1.297 \\
(1.093 \\
1.539)\end{array}$ & 0.003 & $\begin{array}{l}1.224 \\
(1.029, \\
1.457)\end{array}$ & 0.023 \\
\hline $\begin{array}{l}\text { Quartile } \\
4\end{array}$ & $447 / 3143$ & $\begin{array}{l}2.150 \\
(1.829, \\
2.528)\end{array}$ & $<0.001$ & $\begin{array}{l}1.327 \\
(1.108, \\
1.588)\end{array}$ & 0.002 & $\begin{array}{l}1.363 \\
(1.125, \\
1.652)\end{array}$ & 0.002 \\
\hline $\begin{array}{l}\text { Per 1 SD } \\
(0.58)\end{array}$ & $1331 / 12573$ & $\begin{array}{l}1.376 \\
(1.307 \\
1.449)\end{array}$ & $<0.001$ & $\begin{array}{l}1.141 \\
(1.072, \\
1.214)\end{array}$ & $<0.001$ & $\begin{array}{l}1.189 \\
(1.106, \\
1.278)\end{array}$ & $<0.001$ \\
\hline
\end{tabular}

Model 1: Adjusted for age, race, sex.

Model 2: Adjusted for model 1 covariates plus antihypertensive medication, body mass index, coronary heart disease, cholesterol-lowering medication, diastolic blood pressure, diabetes, drinking status (current, former, never), education level (basic, intermediate, advanced education), hypertension, physical activity during leisure time, systolic blood pressure, smoking status (current, former, never), sport during leisure time, stroke.

Model 3: Adjusted for model 2 covariates plus estimated glomerular filtration rate, insulin, low-density lipoprotein cholesterol and total cholesterol.

$H R$ hazard ratio, $\mathrm{Cl}$ confidential interval.

All abbreviations as in Table 1.

Table 3. Risk of incident PAD for TyG index trajectory groups 


\begin{tabular}{|lllllll|}
\hline $\begin{array}{l}\text { TyG index } \\
\text { trajectories }\end{array}$ & $\begin{array}{l}\text { Model 1 } \\
\text { OR }(95 \% \mathrm{Cl})\end{array}$ & $\begin{array}{l}P \\
\text { value }\end{array}$ & $\begin{array}{l}\text { Model 2 } \\
\text { OR }(95 \% \mathrm{Cl})\end{array}$ & $\begin{array}{l}P \\
\text { value }\end{array}$ & $\begin{array}{l}\text { Model 3 } \\
\text { OR }(95 \% \mathrm{Cl})\end{array}$ & $\begin{array}{l}P \\
\text { value }\end{array}$ \\
\hline Moderate-stable & Reference & 1.0 & Reference & 1.0 & Reference & 1.0 \\
\hline Low-increasing & $\begin{array}{l}1.396(1.152, \\
1.691)\end{array}$ & 0.001 & $\begin{array}{l}1.204(0.986, \\
1.469)\end{array}$ & 0.068 & $\begin{array}{l}1.118(0.914, \\
1.368)\end{array}$ & 0.279 \\
\hline $\begin{array}{l}\text { Moderate- } \\
\text { decreasing }\end{array}$ & $\begin{array}{l}2.009(1.648, \\
2.450)\end{array}$ & $<0.001$ & $\begin{array}{l}1.510(1.215, \\
1.877)\end{array}$ & $<0.001$ & $\begin{array}{l}1.507(1.202, \\
1.890)\end{array}$ & $<0.001$ \\
High-decreasing & $\begin{array}{l}\text { 3.184 }(2.480, \\
4.087)\end{array}$ & $<0.001$ & $\begin{array}{l}2.017(1.508, \\
2.697)\end{array}$ & $<0.001$ & $\begin{array}{l}2.314(1.687, \\
3.175)\end{array}$ & $<0.001$ \\
\hline
\end{tabular}

Model 1: Adjusted for baseline age, race, sex.

Model 2: Adjusted for model 1 covariates plus antihypertensive medication, body mass index, coronary heart disease, cholesterol-lowering medication, diastolic blood pressure, diabetes, drinking status, education level (basic, intermediate, advanced education), hypertension, physical activity during leisure time, systolic blood pressure, smoking status, sport during leisure time, stroke.

Model 3: Adjusted for model 2 covariates plus estimated glomerular filtration rate, insulin, low-density lipoprotein cholesterol and total cholesterol.

OR odds ratio, $\mathrm{Cl}$ confidential interval.

All abbreviations as in Table 1.

\section{Figures}




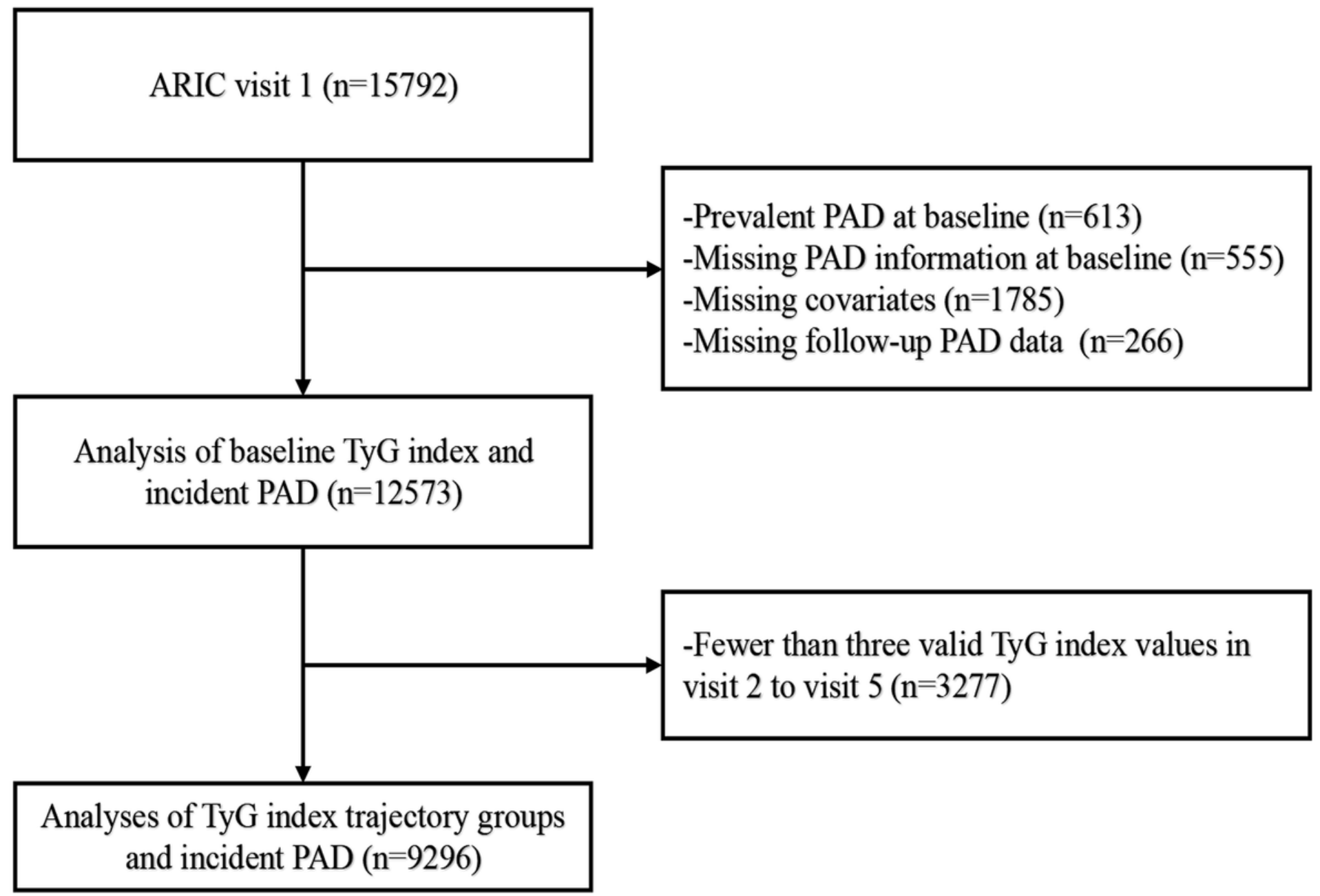

Figure 1

Flowchart for selecting the Atherosclerosis Risk in Communities (ARIC) participants for analysis. 


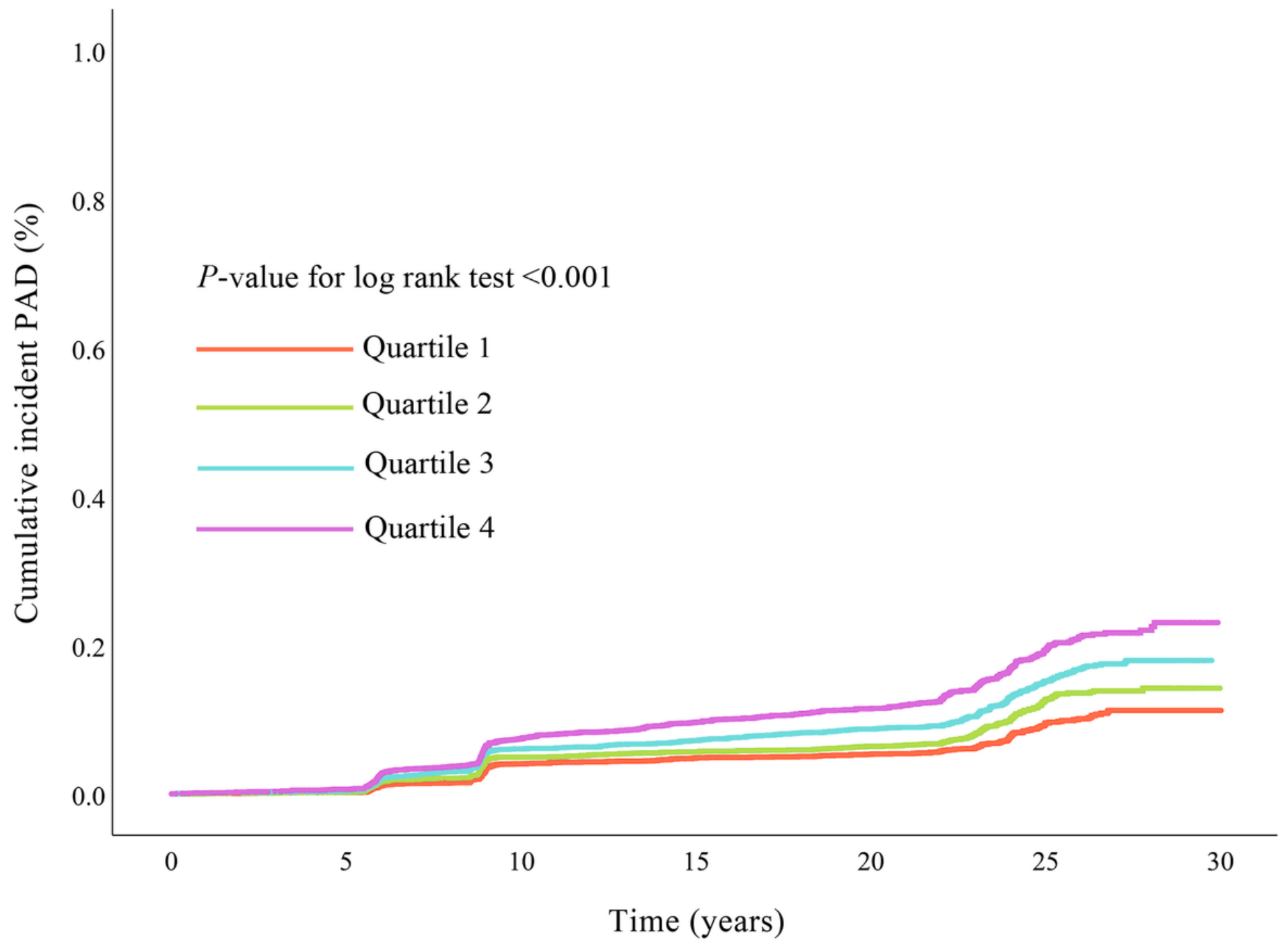

Figure 2

Cumulative incidence of incident PAD by quartiles of baseline TyG index. Cumulative incidence curves are statistically different (log-rank $\mathrm{P}<0.001)$. 


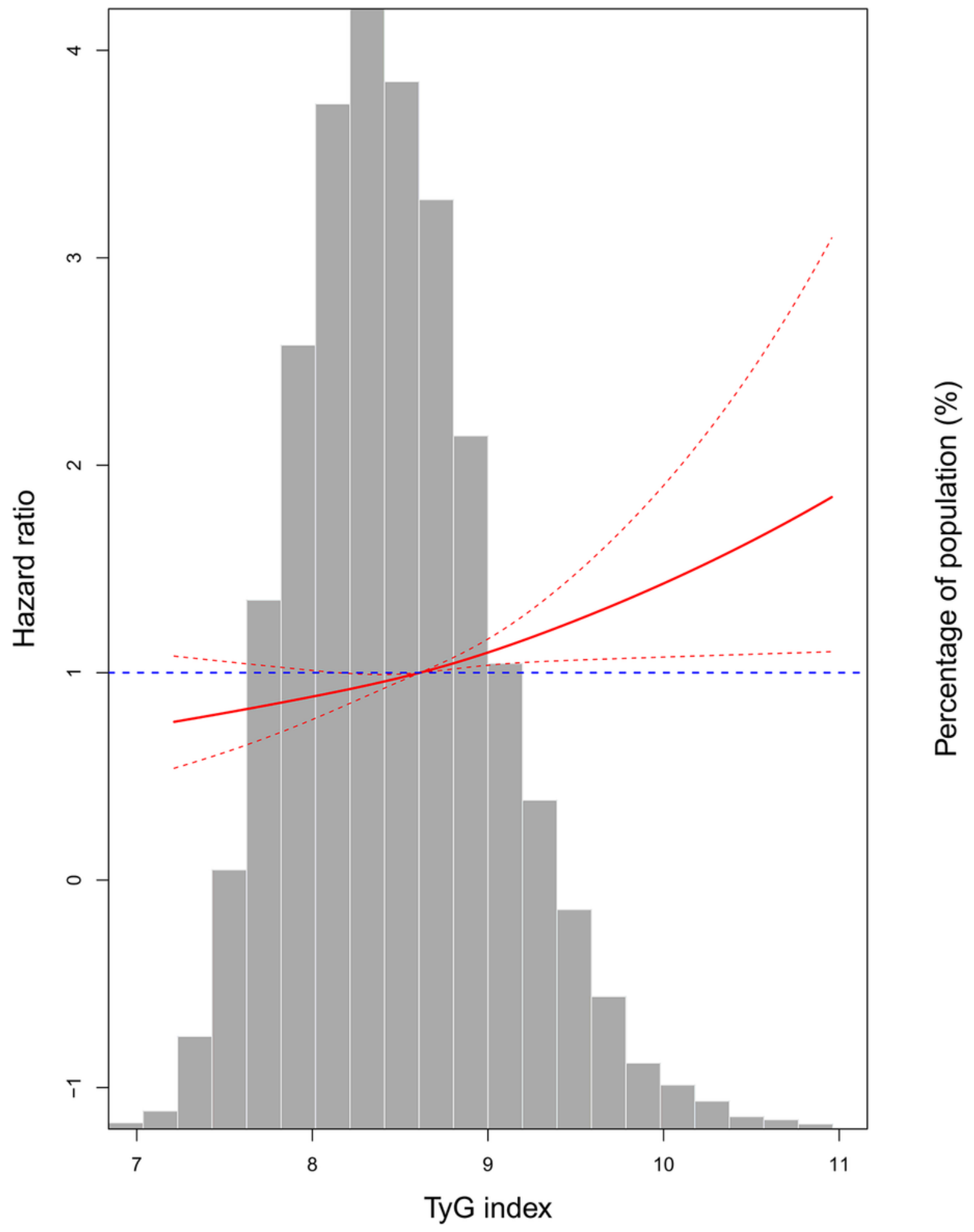

\section{Figure 3}

Adjusted hazard ratios of incident peripheral artery disease (PAD) by baseline triglyceride-glucose (TyG) index. Each hazard ratio was computed with a TyG index level of 8.6 as the reference. The hazard ratio was adjusted for age, sex, race, antihypertensive medication, body mass index, coronary heart disease, cholesterol lowering medication, diastolic blood pressure, diabetes, drinking status, education level, hypertension, physical activity during leisure time, systolic blood pressure, smoking status, sport during 
leisure time, stroke, estimated glomerular filtration rate, insulin, low-density lipoprotein cholesterol and total cholesterol. Red solid line represents the hazard ratio of TyG index across the whole range. Red dotted lines represent the $95 \% \mathrm{Cl}$. Blue dotted line is the reference line as hazard ratio $=1$. Histograms represent the frequency distribution of TyG index.

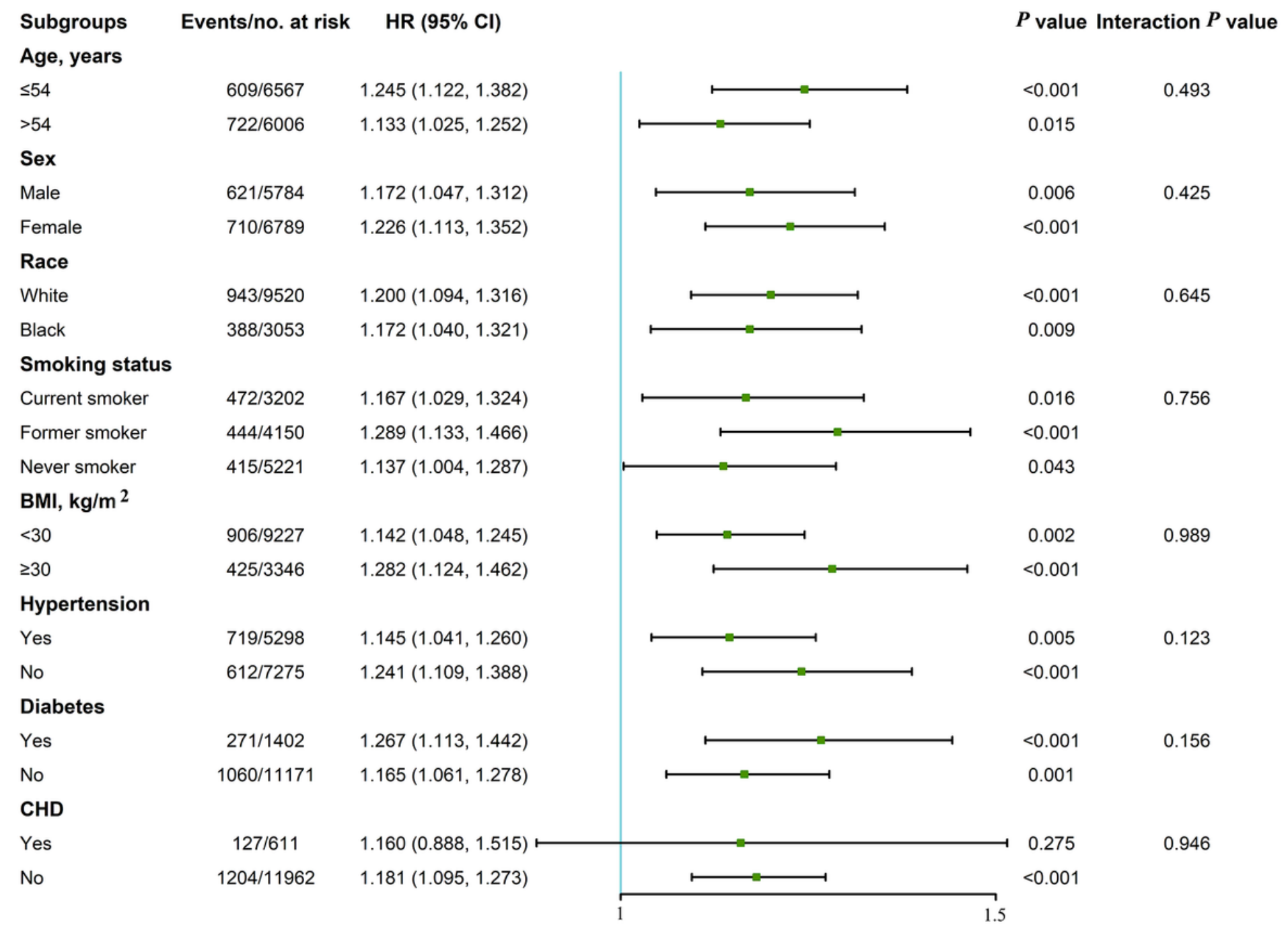

\section{Figure 4}

Subgroup analysis of the association between baseline TyG index and incident PAD. Cox regression after adjustment for antihypertensive medication, cholesterol-lowering medication, coronary heart disease, diastolic blood pressure, drinking status, education level, hypertension, physical activity during leisure time, systolic blood pressure, sport during leisure time, stroke, estimated glomerular filtration rate, insulin, low-density lipoprotein cholesterol and total cholesterol was performed in subgroups according to age ( $\leq 54$ or $>54$ years), gender (male or female), race (White or black), smoking status (current or former or never), body mass index (BMl; $<30$ or $\geq 30 \mathrm{~kg} / \mathrm{m} 2$ ), hypertension (yes or no), and diabetes (yes or no). 
TyG index trajectory groups (percentage of the population in the group)

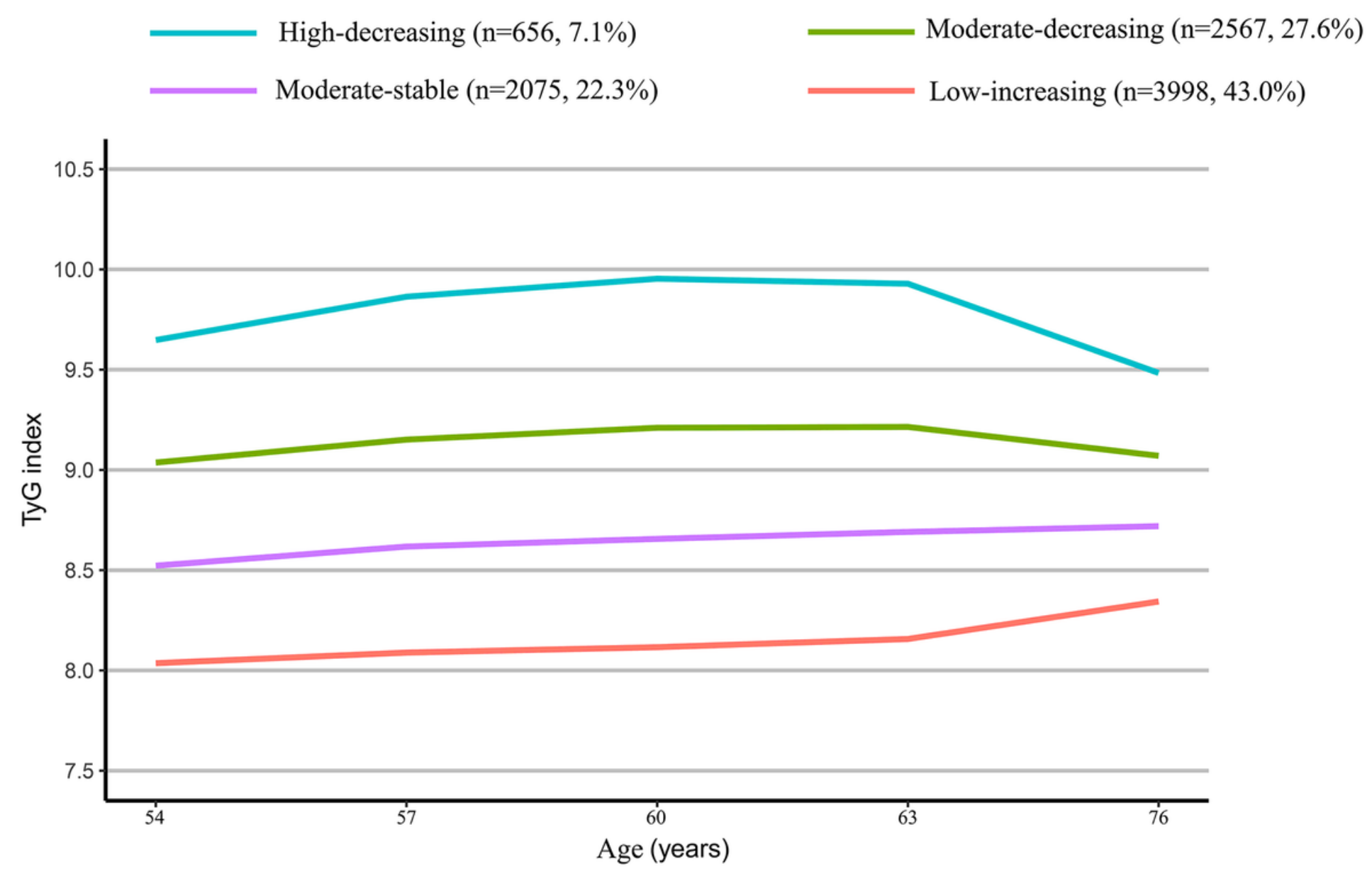

\section{Figure 5}

Trajectories by TyG index in the Atherosclerosis Risk in Communities (ARIC) Study. 

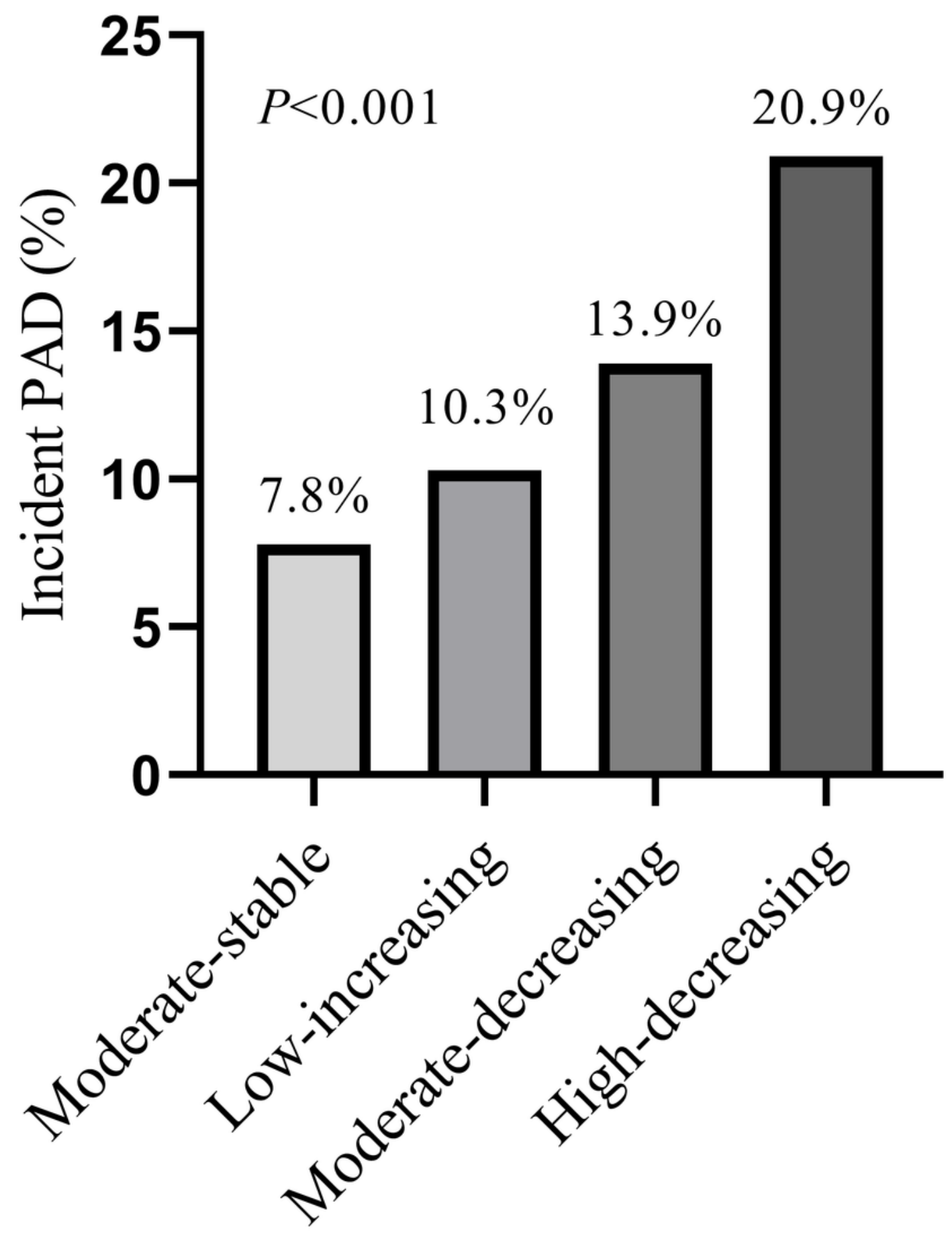

Figure 6

Prevalence of incident PAD across the TyG index trajectory groups.

\section{Supplementary Files}

This is a list of supplementary files associated with this preprint. Click to download. 
- SupplementaryMaterial.docx

Page 24/24 